\title{
Efficiency-Cost Parametric-Analysis of a Three- Phase Wireless Dynamic Charging System for Electric Vehicles
}

\author{
Van-Binh Vu, Member, IEEE, Mohamed Dahidah, Senior Member, IEEE and Volker Pickert, \\ Member, IEEE
}

\begin{abstract}
Wireless dynamic charging (WDC), while the vehicle is in motion, perceived as an enabling technology to mass roll-out of electric vehicles. High output power with minimized pulsation (ripple) is the key-success for such a technology. This paper is presenting a parametric-analysis procedure, aiming at providing design tools for coupler coils of three-phase WDC to achieve specific efficiency and cost requirement. A general design procedure is proposed for a high power WDC system supported by extensive analytical analysis and finite-element analysis (FEA) simulations for wide ranges of transmitter and receiver dimensions, constrained by the stray fields level limitation. Subsequently, other parameters such as the resonant tank are then recalculated to satisfy the system specification. The calculated efficiency is derived according to the loss model of every single component while a cost model is utilized to determine the associated cost, where the tradeoff between efficiency and cost is obtained by parametric-analysis results. The viability of the proposed procedure is experimentally validated using a 3-kW WDC prototype. The practical results show that the efficiency of $88.2 \%$ is achievable with an air gap of $15 \mathrm{~cm}$ and a normalized cost factor of 0.845 .
\end{abstract}

Index Terms-Wireless Dynamic Charging, Electric Vehicles, Finite-element modeling, Parametric-Analysis.

\section{INTRODUCTION}

$\mathrm{W}$ IRELESS dynamic charging (WDC) of Electric Vehicles (EVs) is intended to inductively deliver power from a stationary primary source to one or multiple EV's on-board batteries while in motions [1-2]. As a result, the battery's weight and volume of EVs can be significantly reduced and the driving range limitation can also be alleviated [3]. WDC helps to remove the technological barrier of battery capacity, which uplifts the penetration of EVs in the market. WDC systems are normally classified as either short-individual transmitters [4][7] (i.e., circular pads in [4]-[5] or double-D (DD) and DD quadrature-based pads in [6]-[7]) or long-track transmitters [8][12] (i.e. classified as ferrite core shapes: W-shape [8]-[9], Ishape [10], S-shape [11], or N-shape [12]) depending on the length of the transmitter module.

Manuscript received July 13, 2021; revised August 28, 2021; accepted November 02, 2021. This work was funded by Newcastle University in the Newcastle-Singapore Research Scholarship scheme. (Corresponding author: Van-Binh $V u$.).

The authors are with the School of Engineering, Merz Court, Newcastle University, Newcastle upon Tyne, NE1 7RU, U.K. (e-mail: V.B.Vu2@ncl.ac.uk; mohamed.dahidah@newcastle.ac.uk; volker.pickert@newcastle.ac.uk).
While the former has the advantages of higher efficiency and lower stray fields level, on the other hand, the latter offers lower cost and less complexity. However, both systems suffer from the output power pulsation problem because of the mutual inductance variation, while the $\mathrm{EV}$ is in motions along the driving direction [10]. To address the power pulsation issue, the concept of using three-phase transmitters [14] or multiphase transmitters [13], [15] have been recently proposed, as shown in Fig. 1. The three-phase transmitters, (i.e., $L_{1}, L_{2}$, and $L_{3}$ ) utilize three copper windings to guarantee a homogeneous mutual magnetic flux for the receiver, $L_{s}$ along the driving direction. This results in a constant induced voltage across the vehicle's receiver and hence a constant output power for charging the EV battery.

A number of factors must be considered when designing a three-phase WDC system. The performance of WDC largely depends on factors that are directly related to the wireless couplers. These include: the length and width of the transmitter module, area and weight of the receiver, air gap between two coils, and level of misalignment, etc. Furthermore, the transmission efficiency heavily depends on the coupling coefficient $k$ between transmitter and receiver as well as the quality factor $Q$ of each coupler. On the other hand, both $k$ and $Q$ depend on the coupler's dimensions, used materials, air gap, and misalignment between the couplers. To effectively design a wireless coupler for the stationary EVs charger, the multiobjective approach has been used in [16]-[19] to evaluate the system performance such as efficiency, stray field exposure, power density, and tolerance under misalignment conditions. Specifically, in [16] the design tradeoff between efficiency and power quality in the forms of Pareto fonts are conducted for the two couplers of circular coils. A multi-objective optimization for designing the low-power IPT charging is presented in [17]. A comprehensive evaluation between the rectangular and double-D coil geometries for high power wireless chargers of $50 \mathrm{~kW}$ are also presented in [18]. The work in [19] extends the multi-objective approach presented in [16]-[18] to compare all conflicting performances (i.e., efficiency, stray field exposure, and misalignment tolerance) of four major coupler topologies.

An equally important factor to consider in a WDC system is the implementation cost [20]-[21]. The initial cost in a WDC system for EVs is significantly higher than the stationary 
wireless charger and the conventional conductive charger. The main driver for the high cost of the charging infrastructure is the primary side, which generally consists of multiple transmitter modules for a particular route. Each module constitutes power inverter, the required resonant networks, and the transmitter coupler. In [20], an optimization algorithm is established to economically allocate the transmitter modules on the given routes as well as to calculate the required capacity for the vehicle's battery. In [21], different lengths of transmitter modules are analyzed to comprehend its impact on the overall system cost. Moreover, the benefits of dynamic charging are quantitatively analyzed in the light of battery size and charging infrastructure. Regarding the efficiency, authors in [22]-[24] concluded that the length of each transmitter sectional track should be limited [22], ideally shorter than a vehicle's length to maximize the coupling factor as well as to avoid unwanted losses and heating [23], [24]. Therefore, a suitable design procedure to minimize the cost while achieving good efficiency is undoubtedly of paramount importance. However, quantitative design and evaluation methods incorporating both, efficiency and cost for WDC systems have not yet been reported in the literature.

Hence, this paper attempted to fill in this research gap by proposing the efficiency-cost parametric-analysis of a threephase WDC system using the finite-element analysis (FEA) tools. The transmitter width $\left(W_{p}\right)$ and the ferrite area $A_{r}$ of the receiver are utilized as varying design factors in the analysis while the length of one transmitter module $T_{l}$ is fixed. $W_{p}$ and $A_{r}$ significantly affect the initial cost not only because of the ferrite cost, but also the cost associated with the construction on the road. The FEA simulation is utilized here to extract the coupling coefficient and quality factors of the transmitters and receiver. Consequently, the resonant network (i.e., capacitors and inductors) is calculated to guarantee the WDC system specifications of output power and current [15]. After that, a loss model is used to determine all component losses in the three-phase system when both $W_{p}$ and $A_{r}$ vary. Accordingly, the cost and efficiency parametric-analysis curves are generated. The results quantitatively indicate how the two performance indices, i.e., efficiency and cost, are in a tradeoff relationship. Besides, the impact of varying $T_{l}$ on the system design and performance is also examined after determining the values of $W_{p}$ and $A_{r}$. The proposed parametric-analysis design is validated by means of simulation and experiment of WDC system with $2.4 \mathrm{~m}$ transmitter module length. Based on the parametric-analysis process and other constraints, such as budget, laboratory safety measures, and availability of space and material; a scaled-down prototype of $3-\mathrm{kW}$ WDC is developed in the lab. The experimental results indicate an efficiency of $88.2 \%$ is achieved with an air gap of $15 \mathrm{~cm}$ and a normalized cost factor of 0.845 . This selection of the cost factor is largely influenced by the availability of resources and budget.

\section{EFFICIENCY-COST PARAMETRIC-ANALYSIS}

\section{A. The three-phase WDC system configuration}

An EV WDC system with the multiphase transmitter [15] is presented in Fig. 1(a). The system consists of adjacent multiple transmitter modules placed along the driving direction of given route. Each transmitter module is energized by a power electronic inverter and the associated resonant network. It is worth mentioning that all transmitter modules are independent from each other, and each module is switched ON only once it is aligned with the on-board receiver. After determining the values of $W_{p}$ and $A_{r}$, the impact of varying $T_{l}$ is analyzed in section IV.B. Each transmitter module is composed of three windings arranged in a novel configuration to achieve constant output power at the receiver side [15]. It is worth mentioning that this paper focuses only on the three-phase transmitter WDC system. Fig. 1(b) presents the circuit diagram of the three-phase WDC system for one transmitter module. This includes a threephase inverter, primary and secondary LCC resonant networks, the coupler coils, and the rectifier. At the secondary side, this work considers only a single receiver with a resistive load emulating the battery impedance for simplicity.

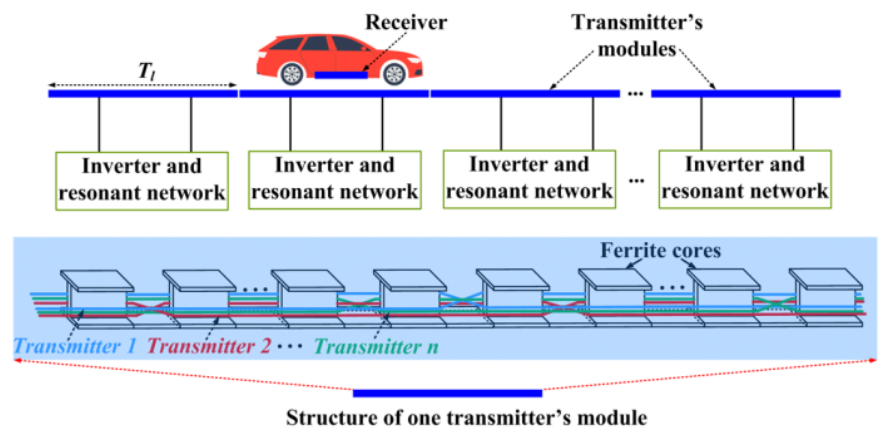

(a)

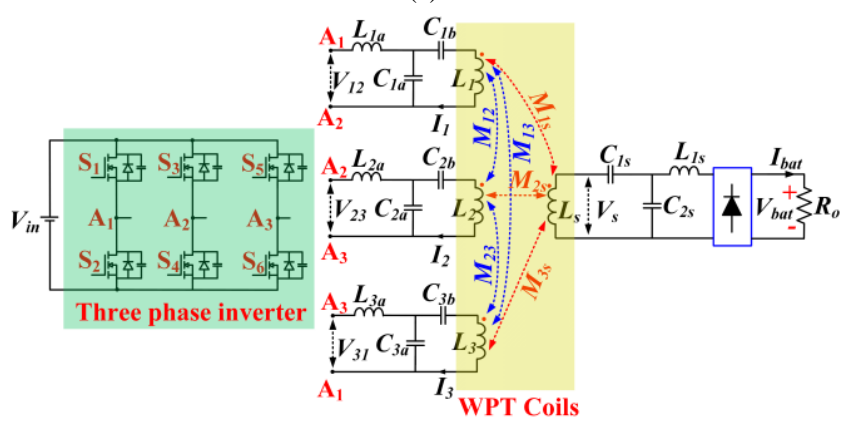

(b)

Fig. 1. (a) Overall structure of the multiphase WDC system [15]. (b) The electrical schematic of a three-phase transmitter module WDC system.

\section{B. Parametric-analysis method}

Fig. 2 illustrating the WDC couplers, where the three-phase transmitters are shown in Fig. 2(a) while the receiver with a rectangular shape is depicted in Fig. 2(b). The efficiency-cost parametric-analysis process is outlined in Fig. 3 which mainly focuses on the coupler's parameters. There are eight consecutive steps in the parametric-analysis process. Step 1 begins with the definition of the three-phase WDC charger specification including the output current, voltage, air gap, operating frequency, and stray fields level limitation, etc. The specifications of WDC system can be found in Table I. The specified power of $3 \mathrm{~kW}$ is selected for the demonstrator due to this being the smallest possible scale for the EV charging level 1. Simultaneously the system must follow the design rules to 
provide a constant output power when the receiver is in motion along the driving direction [15].

Step 2 defines the transmitter's parameter parametric analysis, where several transmitter parameters are initialized, which includes the ferrite thickness, the pole distance, and number of turns. To reduce the unwanted conduction losses and heating, the length of each transmitter's module $T_{l}$ should be shorter than a vehicle's length, which is around 3-4 $\mathrm{m}$ [23]-[24]. Also, the length of each transmitter's sectional track should be similar to the receiver's length (i.e., $0.6 \mathrm{~m}$ ) to maximize the coupling coefficient between two sides. Considering the available ferrite dimension to assemble the transmitter's module, the length of one transmitter's module is selected as $2.4 \mathrm{~m}$ in this work. Noting that each transmitter module consists of four sectional tracks connected in series with a length of 0.6 $\mathrm{m}$, each. Thickness values (i.e., $T_{b}, T_{p p}$, and $T_{p}$ ) are selected such that saturation is avoided, therefore, these values have a minimal effect on the coupling factor $k$. The pole distances $d_{p}$, on the other hand, has a significant impact on $k$ [15]. To simplify the parametric-analysis process, both thickness values and $d_{p}$ are kept constant in this work. At the secondary side, the length and width of the receiver pad, $l_{r}$ and $w_{r}$ are designed to match with the selected $d_{p}$ for a maximum coupling coefficient under different lateral misalignment conditions. As a result, a receiver with dimensions of $0.60 \mathrm{~m} \times 0.60 \mathrm{~m}$ is implemented.

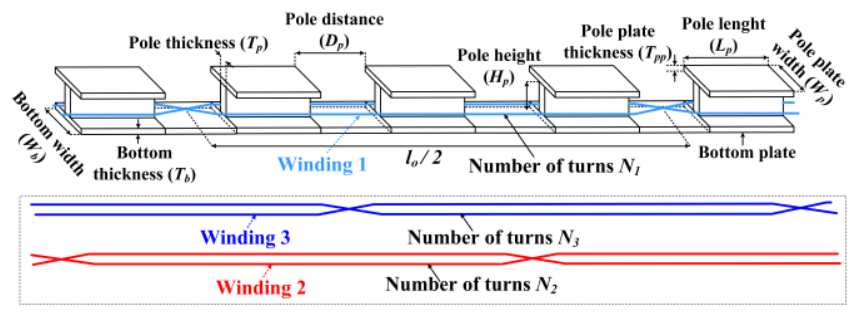

(a)

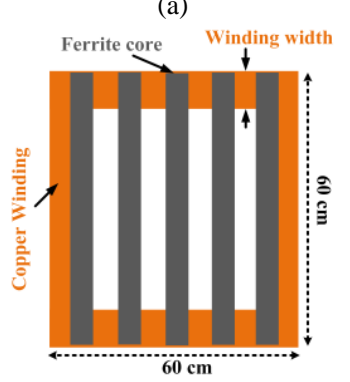

(b)

Fig. 2. The three-phase WDC coupler with detailed parameters. (a) Transmitter side. (b) Receiver side.

TABLE I

SPECIFICATIONS OF THE WDC PROTOTYPE SYSTEM

\begin{tabular}{|c|c|c|}
\multicolumn{2}{c}{ SPECIFICATIONS OF THE WDC PROTOTYPE SYSTEM } \\
\hline \multirow{2}{*}{ Symbol } & Parameters & Value \\
\hline$V_{i n}$ & Input DC voltage & $400 \mathrm{~V}$ \\
\hline$I_{o}$ & Rated output current & $6.5 \mathrm{~A}$ \\
\hline$V_{o}$ & Rated output voltage & $450 \mathrm{~V}$ \\
\hline$P_{o}$ & Rated output power & $3 \mathrm{~kW}$ \\
\hline$D$ & Normal air gap & $15 \mathrm{~cm}$ \\
\hline$f$ & Switching frequency & $85 \mathrm{kHz}$ \\
\hline & $\begin{array}{c}\text { Stray fields level } \\
\text { limitation }\end{array}$ & $\begin{array}{c}<27 \mu \mathrm{T} \text { @ } 1 \mathrm{~m} \mathrm{far} \text { away from } \\
\text { the transmitter }\end{array}$ \\
\hline
\end{tabular}

Furthermore, a suitable choice of the receiver coil shape can help to improve the power transfer in the WDC system [32]. Table II summarizes all fixed parameters of the coupler coils. Further analysis and explanation for the selection of Table II parameters can be referred to [15].

Once all fixed parameters are obtained, step 3 defines the range and increment of $W_{p}$ and $A_{r}$ according to Table III. There are 9 and 7 operating points of $W_{p}$ and $A_{r}$, respectively. This creates a look-up table with 63 combinations from two variables, i.e., $\left(W_{p}, A_{r}\right)=\left(0.05 \mathrm{~m}, 0 \mathrm{~m}^{2}\right),\left(0.07 \mathrm{~m}, 0 \mathrm{~m}^{2}\right), \ldots$, $\left(0.21 \mathrm{~m}, 0 \mathrm{~m}^{2}\right),\left(0.05 \mathrm{~m}, 0.06 \mathrm{~m}^{2}\right),\left(0.07 \mathrm{~m}, 0.06 \mathrm{~m}^{2}\right), \ldots,(0.21 \mathrm{~m}$, $\left.0.06 \mathrm{~m}^{2}\right), \ldots,\left(0.05 \mathrm{~m}, 0.36 \mathrm{~m}^{2}\right),\left(0.07 \mathrm{~m}, 0.36 \mathrm{~m}^{2}\right), \ldots,(0.21 \mathrm{~m}$, $0.36 \mathrm{~m}^{2}$ ). The FEA model is set up in step 4 by using the Ansys Maxwell 3D for each combination to compute their electromagnetic performance as shown in Fig. 4. To reduce the computation time, the Litz wire winding is created by a 3D lumped model with uniform current density. Next, the corresponding self-inductances of the transmitter and the receiver (i.e., $L_{1}, L_{2}, L_{3}, L_{s}$ ) and the mutual inductance (i.e., $M_{l s}$, $M_{2 s}, M_{3 s}$ ) are obtained using FEA simulation as depicted in Step 4. At this point, the coupling coefficient $k$ (i.e., $k=\frac{M_{1 s}}{\sqrt{L_{1} L_{s}}}$ ) and the quality factor $Q$ of the coils (i.e., $Q_{1}=\frac{\omega L_{1}}{r_{1}}, Q_{s}=\frac{\omega L_{s}}{r_{s}}$ ) are then calculated. This process is repeated for all the 63 combinations. After step 4, step 5 calculates $L_{i a}, C_{i a}, C_{i b}(i=$ $1,2,3)$ in the primary side and $L_{1 s}, C_{1 s}, C_{2 s}$ in the secondary side according to the coupler parameters such as self-inductances $\left(L_{1}, L_{2}, L_{3}\right.$, and $\left.L_{S}\right)$, mutual inductances $\left(M_{1 s}, M_{2 s}, M_{3 s}\right)$, crossing inductances $\left(M_{12}, M_{23}, M_{13}\right)$ and system specification. Step 5's calculation is based on [13]-[15] and are not repeated in this paper for simplicity.

TABLE II

FIXED PARAMETERS OF THE COUPLER

\begin{tabular}{|c|c|c|}
\hline \multirow{2}{*}{ Symbol } & Parameters & Value \\
\hline & Transmitter's parameters & \\
\hline$T_{p}$ & Pole thickness & $1.5 \mathrm{~cm}$ \\
\hline$T_{b}$ & Bottom thickness & $0.5 \mathrm{~cm}$ \\
\hline$T_{p p}$ & Pole plate thickness & $0.5 \mathrm{~cm}$ \\
\hline$H_{p}$ & Pole height & $4 \mathrm{~cm}$ \\
\hline$D_{p}$ & Pole distance & $7.5 \mathrm{~cm}$ \\
\hline$T_{l}$ & $\begin{array}{c}\text { Length of one transmitter } \\
\text { module }\end{array}$ & $2.4 \mathrm{~m}$ \\
\hline$I_{l}, I_{2}, I_{3}$ & $\begin{array}{c}\text { Rated Transmitter current for } \\
\text { each phase (RMS) }\end{array}$ & $20 \mathrm{~A}$ \\
\hline$N_{l}, N_{2}, N_{3}$ & Number of turns for each phase & 4 \\
\hline & Receiver's parameters & \\
\hline$R_{l}$ & Receiver's length & $60 \mathrm{~cm}$ \\
\hline$R_{w}$ & $\begin{array}{c}\text { Receiver's width } \\
\text { Receiver's winding's width }\end{array}$ & $60 \mathrm{~cm}$ \\
\hline
\end{tabular}

In step 6, a loss model is developed to determine the total losses of the whole circuit, which calculates the conduction and switching losses of the power electronic inverter, ferrite, and copper losses of resonant inductors and the coupler as well as the conduction losses of capacitors and the diode bridge rectifier. Subsequently, the efficiency is calculated for all the 63 different operating points. The details of the loss calculation are presented in Section III.

After determining the efficiency, the total cost of the system, which is divided into two parts, fixed and variable costs; is 
obtained by a cost model. Fixed costs include every component apart from ferrite cores in both transmitters and receiver, and copper wires of the transmitters. Furthermore, for simplicity, the cost of all resonant capacitors and inductors are assumed to be unchanged despite the fact that their values might slightly change for different operating points. It is because that the cost difference is significantly small compared to the total cost, therefore the cost difference can be negligible. Variable costs are associated with two above listed components for transmitters and receiver. In this work, the costs are illustrated based on the system specifications of Table I, where the detailed calculation is reported in Section IV.

Finally, the relationship of efficiency and the implementation costs are presented according to two variables of $W_{p}$ and $A_{r}$ in the proposed WDC system. From the results, a suitable design can be chosen for the realization of a prototype, considering the tradeoff between efficiency and cost as well as the budget and the availability of space and materials for implementation.

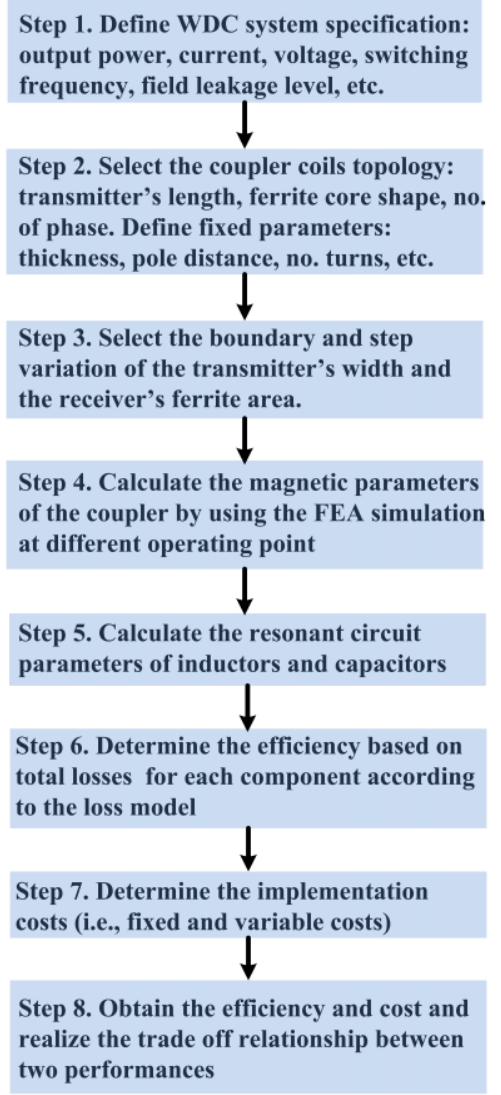

Fig. 3. Parametric-analysis process for one module of the three-phase WDC system.
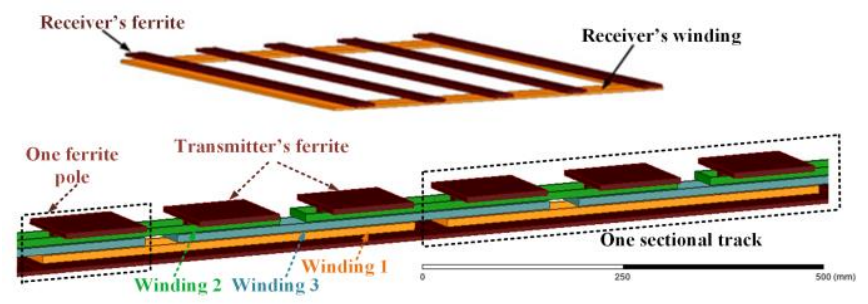

Fig. 4. Simulation model of the couplers using the fixed parameters in Table II and the variable parameters in Table III.
TABLE III

VARIABLE PARAMETERS OF THE COUPLER
\begin{tabular}{|c|c|c|c|c|}
\hline Parameters & Min & Max & Increment & \# Points \\
\hline$W_{p}$ & $0.05 \mathrm{~m}$ & $0.21 \mathrm{~m}$ & $0.02 \mathrm{~m}$ & 9 \\
\hline$A_{r}$ & $0 \mathrm{~m}^{2}$ & $0.36 \mathrm{~m}^{2}$ & $0.06 \mathrm{~m}^{2}$ & 7 \\
\hline
\end{tabular}

\section{THE SYSTEM EFFICIENCY CALCULATION}

The data obtained from Step 4 is used to calculate the parameters of the resonant tank circuit in Step 5. After that, the losses in the coupler and whole prototype are extracted. These include losses associate with the coupler's coils (i.e., $L_{1}, L_{2}, L_{3}$, and $L_{s}$ ), inverter losses (i.e., conduction and switching losses), resonant circuit components (i.e., $L_{1 a}, L_{2 a}, L_{3 a} L_{1 s}, C_{1 a}, C_{1 b}, C_{2 a}$, $C_{2 b}, C_{3 a}, C_{3 b}, C_{l s}$, and $C_{2 s}$ ), and the diode bridge rectifier. The following subsections explain the loss model and the efficiency calculation.

\section{A. Losses of wireless couplers}

The coupler losses include the copper losses $\left(P_{c u}\right)$ and the ferrite losses $\left(P_{f e}\right) . P_{c u}$ constitutes of $P_{s e}$ losses (caused by skin effects) and $P_{p e}$ losses (caused by proximity effect). The equations of $P_{s e}, P_{p e}$, and $P_{c u}$ are given as follows (1-3) based on [16-19].

$$
\begin{gathered}
P_{s e}=n_{s t} \cdot R_{d c} \cdot F_{R}(f) \cdot\left(\frac{I_{\text {peak }}}{n_{s t}}\right)^{2} \\
P_{p e}=n_{s t} \cdot R_{d c} \cdot G_{R}(f) \cdot\left(H_{e}^{2}+\frac{I_{\text {peak }}^{2}}{2 \pi^{2} d_{a}^{2}}\right) \\
P_{c u}=P_{s e}+P_{p e}
\end{gathered}
$$

In (1), $n_{s t}$ denotes the number of strands in the Litz wire (i.e., 600 strands in the prototype of this work), $R_{d c}$ is the DC resistance per unit length of a single strand Litz wire, $I_{\text {peak }}$ is the transmitter peak current and $F_{R}(f)$ is a frequencydependent factor of skin effect [25]. In (2), $d_{a}$ is the outer diameter of the single strand Litz wire, $G_{R}(f)$ depicts a frequency dependent factor of proximity effect while $H_{e}$ represents the external magnetic field penetrating the windings. The coefficients of $F_{R}(f)$ and $G_{R}(f)$ are obtained from [25] while $R_{d c}$ is attained from the manufacturer datasheet [28]. The ferrite core losses can be obtained based on the Steinmetz equation where, $V$ is the region in three-dimensional space [19].

$$
P_{f e}=\iiint_{0}^{V} k_{s} f^{\alpha} B^{\beta} d V
$$

In this paper, core material of PC47 from TDK Electronics is used [26]. The Steinmetz parameters $k_{s}, \alpha$, and $\beta$ of the PC47 material are 5.78, 1.35, and 2.46, respectively.

\section{B. Losses of the inverter}

The inverter losses consist of conduction and switching losses. The total conduction loss is the sum of losses occurred in every switch $S_{i}(i=1,6)$. The losses of $S_{1}, S_{2}$, and $S_{3}$ are equal to that of $S_{4}, S_{5}$, and $S_{6}$, respectively. Therefore, the total conduction loss of the three-phase inverter, $P_{c d}$ can be expressed as follows:

$$
P_{c d}=2 R_{d s}\left(I_{d s 1}^{2}+I_{d s 2}^{2}+I_{d s 3}^{2}\right)
$$

Where $R_{d s}$ is the on-state resistance of each switch while $I_{d s i}(i=1,3)$ is the RMS value of the drain current of switch 
$S_{i}$. On the other hand, the total switching loss in the three-phase inverter can be represented as follows:

$$
P_{S w}=\sum_{i=1}^{6} \frac{1}{2} V_{D S} I_{d s i} f\left(t_{o n i}+t_{o f f i}\right)
$$

In (6), $t_{\text {oni } i}$ and $t_{\text {off } i}$ are the turn-on and turn-off periods of MOSFET switch $S_{i}(i=1,6)$ respectively, $f$ is the switching frequency while $V_{D S}$ is equal to the input DC voltage $V_{i n}$. Eq. (6) is generally expressing the switching losses during both turn-on and turn-off periods where the loss caused during turnon period is far higher than that of the turn-off period. However, in the experiment, turn-on zero-voltage switching (ZVS) is achieved for the whole range of operation, leading to a zero turn-on switching loss. Therefore, the switching losses $P_{S w}$ are very small and neglected in the analysis for simplicity. From (5) and (6), the total losses of the inverter, $P_{i n v}$ can be calculated by (7), where $P_{s w}=0$ :

$$
P_{i n v}=P_{c d}+P_{s w}
$$

\section{Losses of resonant networks}

The losses associated with the resonant networks can be classified into $P_{i n d}$, losses of the resonant inductors (i.e., $L_{l a}$, $L_{2 a}, L_{3 a}$, and $L_{1 s}$ ) and $P_{c a}$, the resonant capacitors (i.e., $C_{l a}, C_{l b}$, $C_{2 a}, C_{2 b}, C_{3 a}, C_{3 b}, C_{1 s}$, and $\left.C_{2 s}\right) . P_{\text {ind }}$ is determined in the same way as described in Section III.A. On the other hand, the capacitor dielectric loss of each capacitor, $P_{c a i}$ is calculated as follows:

$$
P_{c a i}=\frac{\tan \delta}{2 \pi f C} I_{C}^{2}
$$

The dissipation factor $\tan \delta$ in (8) is selected as $0.08 \%$ at 85 $\mathrm{kHz}$ based on the datasheet from KEMET [27]. $I_{C}$ is the RMS current through each individual capacitor. Each resonant capacitor is constructed using low-ESR capacitors connected in parallel to further reduce the total dielectric loss $P_{c a}$.

\section{Losses of the rectifier}

The conduction loss on the secondary rectifier is calculated using (9), where $V_{f w D}$ and $r_{D}$ are the forward voltage and the on-state resistance of the rectifier's diode.

$$
P_{\text {rec }}=\frac{4 \sqrt{2}}{\pi} V_{f w D} I_{o}+2 r_{D} I_{o}^{2}
$$

All component parameters used to calculate losses are depicted in Table VI in the supplemental file. From the above analysis, the efficiency of the three-phase WDC system, $\eta$ is then obtained by the following equation.

$$
\eta=\frac{P_{\text {out }}}{P_{\text {out }}+P_{c u}+P_{f e}+P_{\text {inv }}+P_{\text {ind }}+P_{c a}+P_{\text {rec }}}
$$

\section{Cost CAlculation}

The cost of a transmitter module includes both the fixed part $C_{f}$ and the variable part $C_{v}$. Therefore, the total cost of the WDC system for a given route is $m\left(C_{f}+C_{v}\right)$, where $m$ is the number of modules. Table IV lists the fixed cost part whereas the variable cost is given by:

$$
C_{v}=E_{p} W_{p} N_{p} T_{l}+E_{w} l_{t}+E_{A} A_{r}
$$

Where $E_{p}$ is the cost per unit length of transmitter's top and bottom ferrite plates $(£ / \mathrm{m}) ; W_{p}$ is the transmitter width (m); $N_{p}$ is the number of ferrite poles per meter (illustrated in Fig. 4); $T_{l}$ is the length of one module; $E_{w}$ is the cost per unit of transmitter winding $(£ / \mathrm{m}) ; l_{t}$ is the total length of the transmitter winding

\begin{tabular}{|c|c|c|c|}
\hline Component & Quantity & $\begin{array}{c}\text { Manufacturer/ } \\
\text { Provider }\end{array}$ & Price (£) \\
\hline $\begin{array}{c}\text { Transmitter ferrite } \\
\text { poles }\end{array}$ & 24 pieces & TDK Electronics & 143 \\
\hline $\begin{array}{l}\text { Receiver's copper } \\
\text { winding }\end{array}$ & $20 \mathrm{~m}$ & Osco (UK) & 100 \\
\hline PCB Connectors & 10 & RS Components Ltd & 15 \\
\hline DSP Controller & 1 & Texas Instrument & 505 \\
\hline Gate driver & 3 & Cree Inc. & 150 \\
\hline Inverter & 1 & Cree Inc. & 535 \\
\hline PCB circuits & 2 & $\begin{array}{c}\text { Newbury Electronics } \\
\text { (UK) }\end{array}$ & 55 \\
\hline Protection relay & 2 & Schneider Electric & 98 \\
\hline $\begin{array}{l}\text { Resonant } \\
\text { capacitors }\end{array}$ & 32 & Kemet & 80 \\
\hline Resonant inductors & 4 & $\begin{array}{c}\text { TDK Electronics } \\
\text { (ferrite core only) } \\
\text { Osco (Litz wire only) }\end{array}$ & 32 \\
\hline Rectifier diodes & 4 & $\begin{array}{c}\text { Vishay } \\
\text { Semiconductors }\end{array}$ & 33.88 \\
\hline Filter capacitor & 2 & EPCOS (Ger.) & 12.3 \\
\hline Total fixed cost & & & 1759.2 \\
\hline
\end{tabular}
(meters); $E_{A}$ is the cost per unit size of receiver's ferrite $\left(\mathfrak{f} / \mathrm{m}^{2}\right)$; $A_{r}$ is the total area of receiver's ferrite $\left(\mathrm{m}^{2}\right)$. In this work, $N_{p}$ is designed as 5 per meter while $T_{l}$ is selected as $2.4 \mathrm{~m}$.

TABLE IV

THE FIXED COST PARTS

The variable cost $C_{v}$ depicted in Fig. 5 is calculated using (11), for different values of $W_{p}$ and $A_{r}$. As mentioned earlier, the total transmitter windings $l_{t}$ is proportional with $W_{p}$. Each combination between $W_{p}$ and $A_{r}$ generates a different value for $C_{v}$. However, if ferrite material is not used in the receiver design, then $A_{r}$ is equal zero.

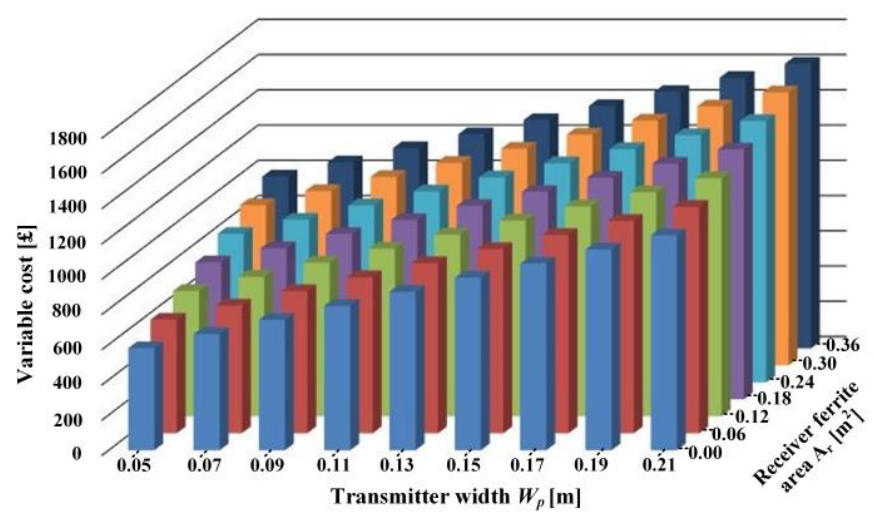

Fig. 5. The variable cost part with different values of $W_{p}$ and $A_{r}$.

For clearer presentation, the normalized cost is defined in (12), which is used instead of the real cost. A normalized cost is the ratio of one design's cost to the maximum one. In (12) the fixed cost $C_{f}=£ 1759.2$ while $C_{v_{-} \max }=£ 1615 . C_{v_{-} \max }$ is associated with $W_{p}=0.21 \mathrm{~m}$ and $A_{r}=0.36 \mathrm{~m}^{2}$. Nevertheless, the variable cost, $C_{v}$ varies according to Fig. 5 .

$$
\text { Normalized cost }=\frac{C_{f}+C_{v}}{C_{f}+C_{v_{-} \max }}
$$




\section{PARAMETRIC-ANALYSIS RESUlts}

\section{A. Impact of $W_{p}$ and $A_{r}$}

Based on the calculation of efficiency and cost in Section III and IV, the parametric-analysis results of the design examples are illustrated in Figs. 6-8. Specifically, Fig. 6(a) and 6(b) represent the variation of the efficiency $\eta$ and the normalized cost with transmitter's width $W_{p}$ and receiver's ferrite area $A_{r}$. From Fig. 6(a), the efficiency $\eta$ varies proportionally with both $W_{p}$ and $A_{r}$. This is basically because higher values of $W_{p}$ and $A_{r}$ result in an increased coupling coefficient and coil quality factors, leading to a higher transmission efficiency. Moreover, the incremental rate of $\eta$ versus $A_{r}$ is not constant in which the rate is higher at the smaller values (i.e., $0.00 \mathrm{~m}^{2}$ to $0.18 \mathrm{~m}^{2}$ ) and lowers down when $A_{r}$ gradually reaches a maximum value of $0.36 \mathrm{~m}^{2}$. It is noted that $A_{r}=0.00 \mathrm{~m}^{2}$ means no ferrite used in the receiver. A similar pattern is noticed for $W_{p}$ variations. On the other hand, the incremental rate of the cost is more linear with both $W_{p}$ and $A_{r}$ as shown in Fig. 6(b). Hence, the operating points should not be selected when $W_{p}$ and $A_{r}$ are at very high values to minimize the cost.

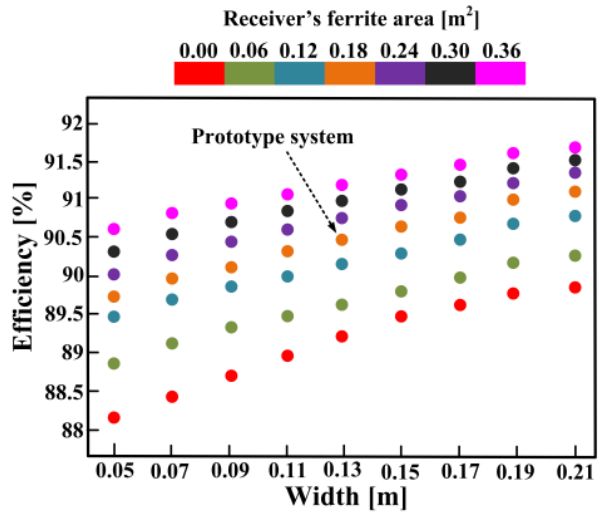

(a)

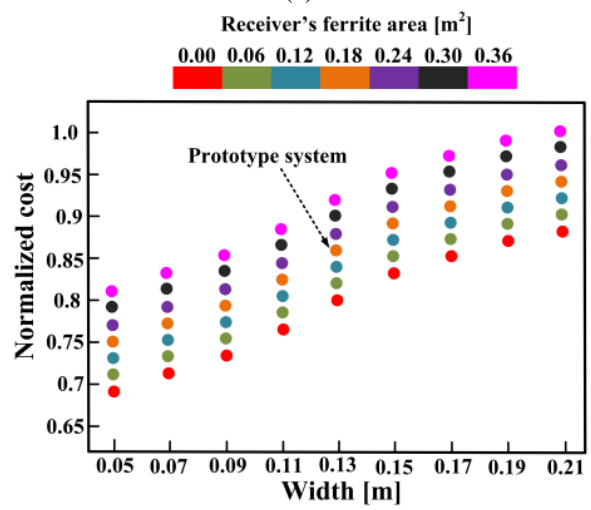

(b)

Fig. 6. (a) Efficiency versus transmitter width and receiver ferrite area, (b) The normalized cost versus transmitter width and receiver ferrite area.

Figs. 7 and 8 present the relationship between both, the efficiency and the associated cost against $A_{r}$ and $W_{p}$. The calculated efficiency varies from $88.23 \%$ to $92.56 \%$ while the normalized cost factor varies between 0.691 to 1 . One can clearly see that there is a tradeoff between the efficiency and the associated cost. A high efficiency can be achieved if largewidth transmitters with a full ferrite receiver are used, implying a high implementation cost. In detail, a maximum transmission efficiency of $92.56 \%$ can be attained if the cost factor is set to 1. A low implementation cost can be achieved if smaller transmitters and a ferrite-less receiver are used; however, a decreased efficiency is occurred simultaneously by means of lower coupling coefficients and coupler's quality factors. This trade-off forms a performance boundary obtained by the design parameters where an increase of one performance factor (cost or efficiency) consequences in a decrease of the other.

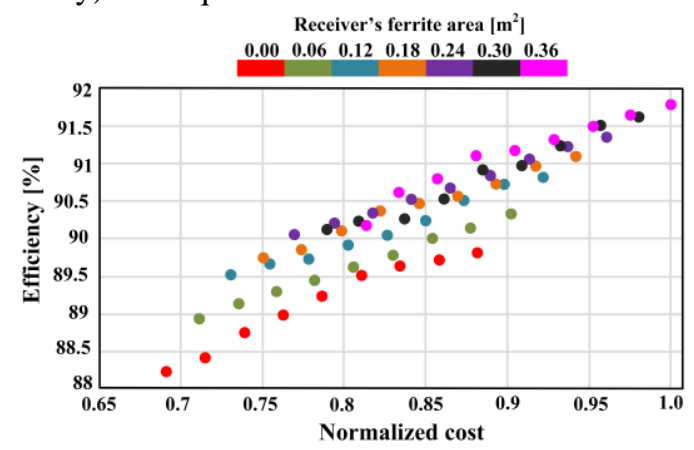

Fig. 7. Efficiency versus normalized cost for different receiver ferrite areas.

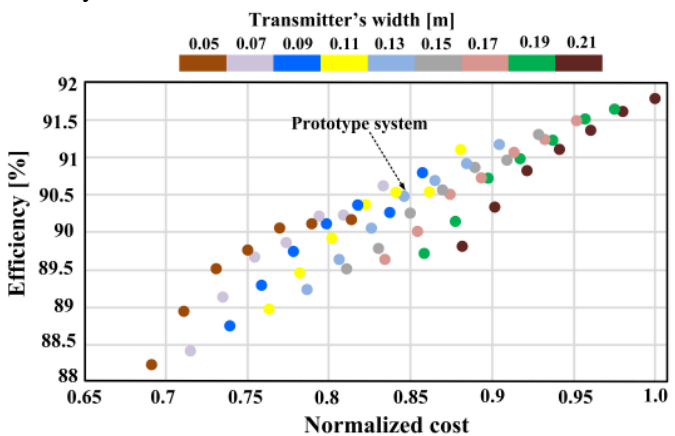

Fig. 8. Efficiency versus normalized cost for different transmitter widths.

Taking the above analysis into consideration, the proposed coupler is selected with $W_{p}$ of $13 \mathrm{~cm}$ and $A_{r}$ of $0.18 \mathrm{~m}^{2}$. This corresponds to a cost factor of 0.845 and an efficiency of $90.49 \%$. This selection is largely influenced by the availability of resources and budget. Future research of utilizing the weighting factor [29] can be considered to have a better selection of the operating point, depending on different system requirement (i.e., whether cost or efficiency is more important).

\section{B. The impact of $T_{l}$ on WDC design}

This section briefly presents the impact of $T_{l}$ on the efficiency and cost of the three-phase WDC system. To simplify the analysis, $W_{p}$ and $A_{r}$ are fixed as $0.13 \mathrm{~m}$ and $0.18 \mathrm{~m}^{2}$, respectively, while $T_{l}$ varies from $1.25 \mathrm{~m}$ to $15 \mathrm{~m}$ with an increment of $1.25 \mathrm{~m}$. As illustrated in Fig. 4, each transmitter module possesses several sectional tracks, and the length of each track should be close to the length of the receiver to maximize the coupling coefficient [22]. Therefore, the length of one sectional track is chosen as $0.6 \mathrm{~m}$ in this work. The relationship between the maximum coupling coefficient $k_{\max }$ and $T_{l}$ is illustrated in Fig. 9(a). Please note that $k_{\max }$ can be only achieved if one transmitter sectional track is aligned with the receiver. It shows that $k_{\max }$ reduced from 0.17 to 0.05 when $T_{l}$ increased from $1.25 \mathrm{~m}$ to $15 \mathrm{~m}$. This can be explained by the 
fact that the maximum mutual inductance $M_{o}$ between one sectional track and the receiver is kept nearly constant when $T_{l}$ varies. However, a longer $T_{l}$ will also result in a higher transmitter's inductances $L_{1}$ that causes $k_{\max }$ to reduce as shown by (13).

$$
k_{\max }=\frac{M_{o}}{\sqrt{L_{1} L_{s}}}
$$

Furthermore, longer $T_{l}$ leads to higher voltage on the series capacitor $C_{1 b}$ and the transmitter's inductance, $L_{1}$ for a given transmitter current. The voltage on the transmitter (i.e., $V_{L 1}$ ) increases with higher $L_{1}$ as depicted in (14). Moreover, the crossing inductance $M_{12}$ between two transmitter windings are also higher when $T_{l}$ increases as can be observed from (15). In details, $k_{12}$ between two transmitter windings is constant however, both $L_{1}$ and $L_{2}$ increase. This results in a higher voltage on $C_{1 b}$ as shown in (16) in which $C_{1 b}=\frac{1}{\omega_{o}^{2}\left(L_{1}-L_{1 a}+M_{12}\right)}$. Fig. 9(b) further shows the simulation results of $V_{L 1}$ and $V_{C 1 b}$ against $T_{l}$. The voltage stress on $C_{1 b}$ is higher than that of $L_{1}$ when $T_{l}$ is greater than $2.5 \mathrm{~m}$. Furthermore, $V_{L 1}$ and $V_{C 1 b}$ can reach to $11.6 \mathrm{kV}$ and $14.1 \mathrm{kV}$, respectively, at the maximum value of $T_{l}$. Therefore, the voltage ratings of $L_{1}$ and $C_{1 b}$ need to be considered carefully in the design for safety concern.

$$
\begin{gathered}
V_{L 1}=\omega_{o} I_{1} L_{1} \\
M_{12}=k_{12} \sqrt{L_{1} L_{2}} \\
V_{C 1 b}=I_{1} \frac{1}{j \omega_{o} C_{1 b}}=I_{1} \omega_{o}\left(L_{1}-L_{1 a}+M_{12}\right)
\end{gathered}
$$

Fig. 9(c) presents the implementation cost against $T_{l}$. for a WDC system with the total length of $1000 \mathrm{~m}$. For simplicity, the primary resonant networks are excluded from the calculation. It can be observed that the total cost is gradually reduced from $£ 1744400$ to $£ 824800$ when $T_{l}$ increases from 2.5 to $15 \mathrm{~m}$. This is because higher number of modules is required for a certain road length with a shorter $T_{l}$, leading to a higher cost. Thus, in addition to $W_{p}$ and $A_{r}$, the transmitter length $T_{l}$ has also a great impact on the implementation cost.

\section{EXPERIMENTAL VERIFICATION}

Building upon the parametric-analysis results of Section V, a laboratory prototype of 3-kW WDC is implemented and tested to validate the design procedure proposed in the work. As previously mentioned, the transmitter width $W_{p}$ is selected as $0.13 \mathrm{~m}$ while the receiver's ferrite core area $A_{r}$ is chosen as 0.18 $\mathrm{m}^{2}$ and other couplers parameters can be found in Table II. The developed prototype is shown in Fig. 10. The operating frequency is $85 \mathrm{kHz}$ to be in line with the current inductive charging standard [30] while the 38-AWG litz wire with 600 strands is chosen to construct both, transmitters and receiver.

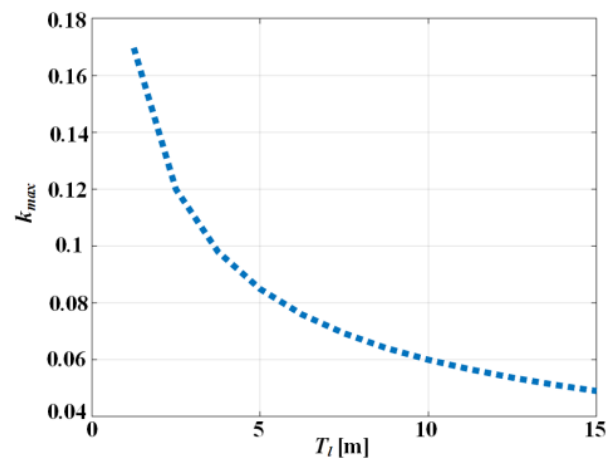

(a)

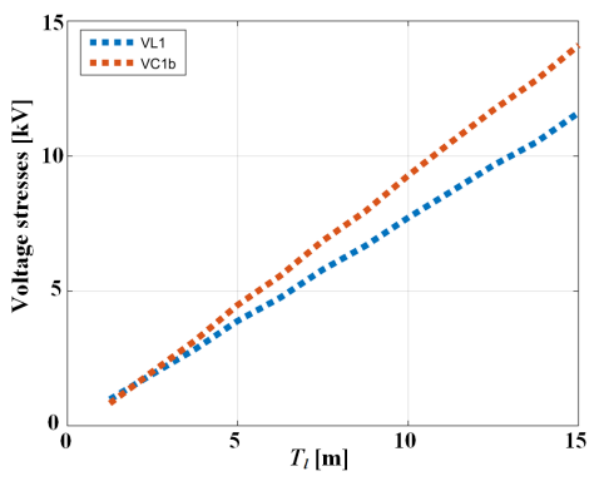

(b)

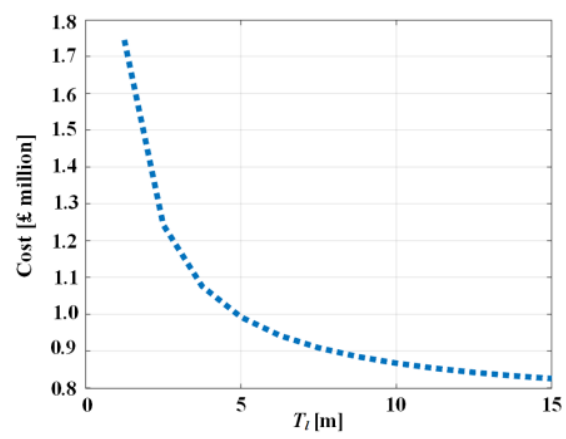

(c)

Fig. 9. Impact of $T_{l}$ on the WDC system performances with $W_{p}=0.13 \mathrm{~m}$ and $A_{r}=0.18 \mathrm{~m}^{2}$. (a) Coupling coefficient $k_{\max }$ against $T_{l}$. (b) Voltage stresses on $L_{1}\left(V_{L 1}\right)$ and $C_{1 b}\left(V_{C 1 b}\right)$ against $T_{l}$. (c) The total cost of 1000-m WDC system against $T_{l}$ (excluding primary resonant networks).

Other parameters of the resonant tank are presented in Table V. The three-phase inverter is built using $\mathrm{SiC}$ MOSFET, C2M0025120D from Cree while SiC-Schottky diodes, HFA50PA60 is chosen for the rectifier. Polypropylene film capacitors from Kemet are selected for the resonant network owing to their low equivalent series resistance and high current carrying capability. Please note that the compensation capacitors $C_{l b}, C_{2 b}$, and $C_{3 b}$ are adjusted from $100.4 \mathrm{nF}$ in simulation to $90.2 \mathrm{nF}$ in experiment for achieving ZeroVoltage-Switching (ZVS) of all inverter's switches. 


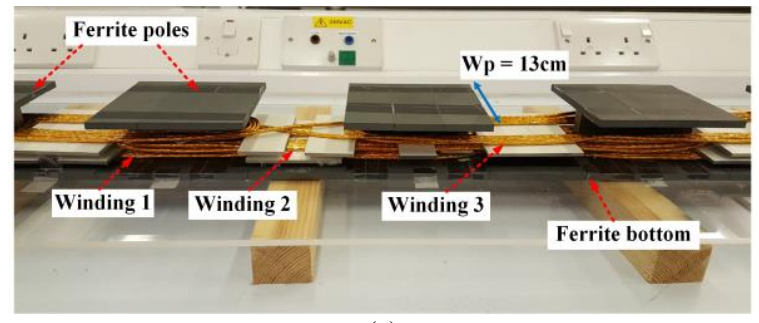

(a)

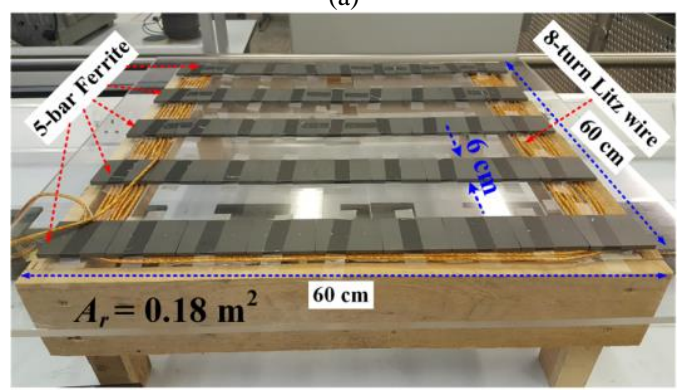

(b)

Fig. 10. Experimental test-rig of the three-phase WDC system. (a) Three-phase transmitters. (b) Receiver.

TABLE V

PARAMETERS OF RESONANT TANK OF WDC SYSTEM

\begin{tabular}{|c|c|c|}
\hline Symbol & Parameters & Value \\
\hline$L_{1}, L_{2}, L_{3}$ & Self-inductance of transmitters & $182 \mu \mathrm{H}$ \\
\hline$M_{12}, M_{13}, M_{23}$ & Crossing mutual inductances & $-46 \mu \mathrm{H}$ \\
\hline$L_{1 a}, L_{2 a}, L_{3 a}$ & Primary additional inductors & $37 \mu \mathrm{H}$ \\
\hline$C_{1 a}, C_{2 a}, C_{3 a}$ & Primary parallel capacitors & $95 \mathrm{nF}$ \\
\hline$C_{1 b}, C_{2 b}, C_{3 b}$ & Primary series capacitors & $90.2 \mathrm{nF}$ \\
\hline$L_{s}$ & Self-inductance of receiver & $118 \mu \mathrm{H}$ \\
\hline$M_{o}$ & Maximum mutual inductance & $12.5 \mu \mathrm{H}$ \\
\hline$L_{1 s}$ & Secondary additional inductor & $35 \mu \mathrm{H}$ \\
\hline$C_{2 s}$ & Secondary parallel capacitors & $100 \mathrm{nF}$ \\
\hline$C_{1 s}$ & Secondary series capacitors & $42.2 \mathrm{nF}$ \\
\hline
\end{tabular}

The key operating waveforms of the WDC system are shown in Fig. 11 under the rated load power of $3 \mathrm{~kW}$ (i.e., load resistance of $70 \Omega$ ). The transmitter currents $I_{1}, I_{2}$, and $I_{3}$ are depicted in Fig. 11(a), where they have the same amplitude but 120 degrees phase-shift between each other. Fig. 11(b) further demonstrates the operation of the WDC system while the receiver is moving. It is noticeable that the output voltage remains nearly constant regardless of the receiver's position. This verifies the good characteristics of the three-phase WDC system in maintaining a constant output power while the receiver is in motions along the transmitter. Fig. $11 \mathrm{~b}$ also shows that the output voltage variation is within $30 \mathrm{~V}$ with the nominal value of $450 \mathrm{~V}$. Noting that one DC/DC converter inserting between the on-board rectifier and battery will be used to smooth the battery voltage. However, this converter is not included and discussed in this paper for simplicity.

The performance of the proposed WDC is further investigated under lateral misalignment with a different air gap, h of 5, 10, and $15 \mathrm{~cm}$. Fig. 12(a) demonstrates that the output power reduces with higher lateral misalignment and air gap between the receiver and the transmitter. For instance, with $15-\mathrm{cm}$ air gap and 30-cm misalignment, then the output power drops to as low as $29 \%$ of the maximum power of $3 \mathrm{~kW}$ at no misalignment. Furthermore, Fig. 12(b) illustrates the system efficiency across a wide range of misalignment and different air gaps. As expected, higher efficiency is obtained with smaller misalignment, as can be seen from the same figure.

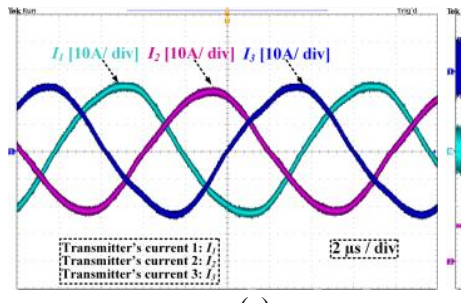

(a)

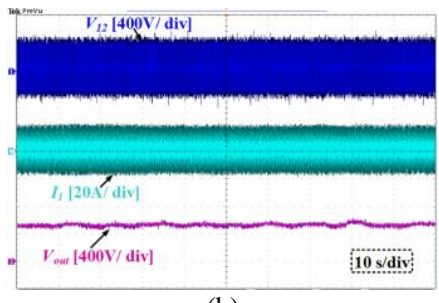

(b)
Fig. 11. Experimental waveforms at rated load of $3 \mathrm{~kW}$. (a) Transmitter currents. (b) output voltage $V_{\text {out }}$ under the receiver's movement along the driving direction.

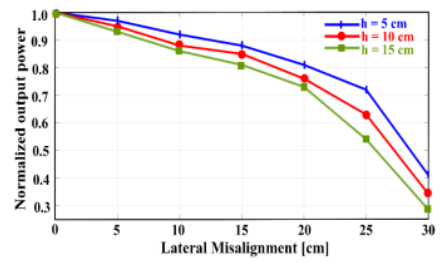

(a)

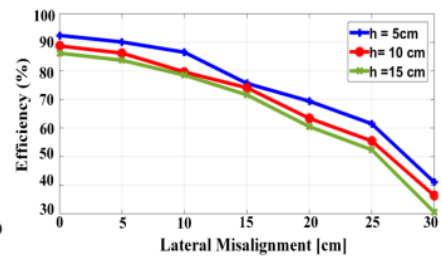

(b)
Fig. 12. System performance under different lateral misalignments and airgaps at the rated load $\left(\mathrm{R}_{\mathrm{o}}=70 \Omega\right.$ ). (a) Normalized output power. (b) Measured output efficiency.

Fig. 13 demonstrates the calculated system loss breakdown while the receiver moves along the driving direction (i.e., from $60 \mathrm{~cm}$ to $120 \mathrm{~cm}$ ). It is noticeable from the loss breakdown graph that the wireless coupler contributes the highest percentage with nearly $60 \%$ while all other parts are below $20 \%$. It is worth noting that the losses of the three-phase system are not significantly changing with receiver movement along transmitter. This is distinctively unique compared to singlephase WDC system in which the loss still exists even when no power is transferred [15]. Fig. 14 shows the measured efficiency of the prototype as a function of the output power and receiver's positions. The measurements demonstrate that a system efficiency of $88.2 \%$ is achieved at the rated power and maintains above $80 \%$ when the power level is more than $1 \mathrm{~kW}$. The analytical loss calculation at $3 \mathrm{~kW}$ output power is $310 \mathrm{~W}$, which is smaller than the measured $354 \mathrm{~W}$ power loss from the experiment. This could be due to the existing parasitic parameters in the printed circuit board, metal objects that surround the test-rig and potential errors in measurement equipment. The experimental bench is surrounded by metal bars for safety reasons and the coupler is placed on the top of a bench that uses a metal frame structure underneath. This causes more losses as the leakage magnetic flux generates the eddy currents in the metal objects [31]. Therefore, there is an increase of total loss in experiments as compared to the calculated results. Moreover, this work focuses only on the analysis of the transmitter coils in the air. Our future studies will investigate further the DWC system's behaviour when the transmitter coils are embedded in the concrete [33]-[34]. 


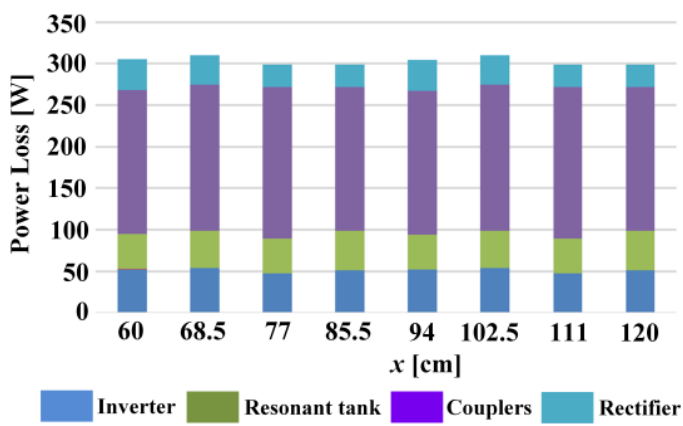

Fig. 13. Calculated losses of the three-phase WDC systems at different positions of receiver along the driving direction.

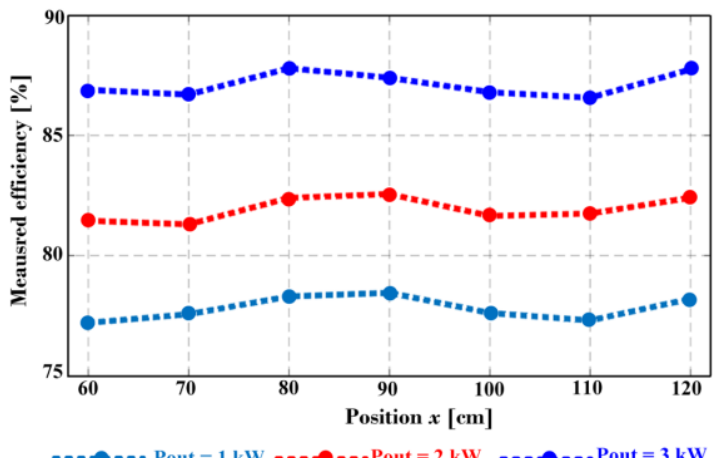

Fig. 14. Measured efficiency as a function of output power level and receiver's positions.

\section{CONCLUSION}

A parametric-analysis based design procedure for three-phase WDC system for EV applications was proposed in this paper. Efficiency and implementation cost were used as an evaluation criterion to identify the best design option for a given scenario. The analysis focused on the coupler design, where extensive FEA simulations were conducted to comprehend various system performances. Three parameters, $W_{p}, A_{r}$, and $T_{l}$ of the coupler were utilized. Loss models for all sub-systems and a normalized cost formula were developed to calculate the overall efficiency and cost of the system, respectively. The results quantitatively indicated the tradeoff between efficiency and cost. A representative three-phase WDC system was then developed in light of the proposed design procedure with cost factor of 0.845 and an efficiency of $90.4 \%$. The measurement shows that the efficiency of the WDC system can achieve $88.2 \%$ at rated load. The experimental result implies a good agreement with analytical calculation one. The method presented in this paper can be used in other wireless charger systems to suitably design the couplers and relevant parameters for satisfying the requirements of both efficiency and cost.

\section{REFERENCES}

[1] G. A. Covic and J. T. Boys, "Modern Trends in Inductive Power Transfer for Transportation Applications," IEEE Trans. Emerg. Sel. Topics Power Electron., vol. 1, no. 1, pp. 28-41, Mar. 2013.

[2] S. Y. Choi et al., "Advances in Wireless Power Transfer Systems for RoadwayPowered Electric Vehicles," IEEE Trans. Emerg. Sel. Topics Power Electron., vol. 3, no. 1, pp. 18-36, Mar. 2015.

[3] C. C. Mi et al., "Modern Advances in Wireless Power Transfer Systems for Roadway Powered Electric Vehicles," IEEE Trans. Ind. Electron., vol. 63, no. 10, pp. 6533-6545, Oct. 2016
[4] J. M. Miller et al., "Demonstrating Dynamic Wireless Charging of an Electric Vehicle: The Benefit of Electrochemical Capacitor Smoothing," IEEE Power Electron. Mag., vol. 1, no. 1, pp. 12-24, March 2014.

[5] J. M. Miller et al., "ORNL Experience and Challenges Facing Dynamic Wireless Power Charging of EV's," IEEE Circuits Syst. Mag., vol. 15, no. 2, pp. 40-53, 2nd Quart., 2015.

[6] G. R. Nagendra, G. A. Covic and J. T. Boys, "Sizing of Inductive Power Pads for Dynamic Charging of EVs on IPT Highways," IEEE Trans. Transport. Electrific., vol. 3, no. 2, pp. 405-417, Jun. 2017.

[7] A. Zaheer et al., "A Dynamic EV Charging System for Slow Moving Traffic Applications," IEEE Trans. Transport. Electrific., vol. 3, no. 2, pp. 354-369, Jun. 2017.

[8] J. Shin et al., "Design and Implementation of Shaped Magnetic-Resonance-Based Wireless Power Transfer System for Roadway-Powered Moving Electric Vehicles," IEEE Trans. Ind. Electron., vol. 61, no. 3, pp. 1179-1192, Mar. 2014.

[9] W. Y. Lee et al., "Finite-Width Magnetic Mirror Models of Mono and Dual Coils for Wireless Electric Vehicles," IEEE Trans. Power Electron., vol. 28, no. 3, pp. 1413-1428, Mar. 2013.

[10] J. Huh et al., "Narrow-Width Inductive Power Transfer System for Online Electrical Vehicles," IEEE Trans. Power Electron., vol. 26, no. 12, pp. 3666-3679, Dec. 2011.

[11] S. Y. Choi, et al., "Ultraslim S-Type Power Supply Rails for Roadway-Powered Electric Vehicles," IEEE Trans. Power Electron., vol. 30, no. 11, pp. 6456-6468, Nov. 2015.

[12] Z. Wang et al., "A Novel Magnetic Coupling Mechanism for Dynamic Wireless Charging System for Electric Vehicles," IEEE Trans. Veh. Tech., vol. 67, no. 1, pp. 124-133, Jan. 2018

[13] V. Vu et al. "A Concept of Multiphase Dynamic Charging System with Constant Output Power for Electric Vehicles," in Proc. IEEE Wireless Power Transfer Conf., pp. 229-233, Jun. 2019.

[14] V. Vu et al. "Comparison of Single and Three phase Dynamic Charging Systems for Electric Vehicles," in Proc. IEEE Wireless Power Transfer Conf., pp. 220-225, Jun. 2019.

[15] V. Vu et al. "A High-Power Multiphase Wireless Dynamic Charging System with low Output Power Pulsation for Electric Vehicles," IEEE Trans. Emerg. Sel. Topics Power Electron., vol. 8, no. 4, pp. 3592-3608, Dec. 2020.

[16] R. Bosshard et al., "Modeling and $\eta-\alpha$-Pareto Optimization of Inductive Power Transfer Coils for Electric Vehicles," IEEE Trans. Emerg. Sel. Topics Power Electron., vol. 3, no. 1, pp. 50-64, March 2015.

[17] S. Bandyopadhyay et al. "Multi-objective optimisation of a 1-kW wireless IPT systems for charging of electric vehicles," in Proc. IEEE Transp. Electrific. Conf. Expo (ITEC), Jun. 2016, pp. 1-7.

[18] R. Bosshard et al. "Comprehensive Evaluation of Rectangular and Double-D Coil Geometry for $50 \mathrm{~kW} / 85 \mathrm{kHz}$ IPT System," IEEE Trans. Emerg. Sel. Topics Power Electron., vol. 4, no. 4, pp. 1406-1415, Dec. 2016.

[19] S. Bandyopadhyay et al. "Comparison of Magnetic Couplers for IPT-Based EV Charging Using Multi-Objective Optimization," IEEE Trans. Veh. Tech., vol. 68, no. 6, pp. 5416-5429, Jun. 2019.

[20] Y. D. Ko and Y. J. Jang, "The Optimal System Design of the Online Electric Vehicle Utilizing Wireless Power Transmission Technology," IEEE Trans. Intell. Transp. Syst., vol. 14, no. 3, pp. 1255-1265, Sep. 2013.

[21] S. Jeong, et al., "Economic Analysis of the Dynamic Charging Electric Vehicle," IEEE Trans. Power Electron., vol. 30, no. 11, pp. 6368-6377, Nov. 2015.

[22] W. Zhang et al., "An Optimized Track Length in Roadway Inductive Power Transfer Systems," IEEE Trans. Emerg. Sel. Topics Power Electron., vol. 2, no. 3, pp. 598608, Sept. 2014.

[23] Covic, et al., "Electric Vehicles - Personal transportation for the future" World Electr. Veh. J. 2010, 4, 693-704.

[24] H. R. Ross, "Roadway-powered electric vehicle system," U.S. Patent 5669 470, Sep. 23, 1997.

[25] J. Mühlethaler, "Modeling and multi-objective optimization of inductive power components," Ph.D. dissertation, Swiss Federal Inst. Technol. Zurich, ETHZ, Zürich, The Switzerland, 2012.

[26] TDK Corporation, "Characteristics of Mn-Zn ferrite", 2021. [Online]. Available: https://product.tdk.com/en/system/files?file=dam/doc/product/ferrite/ferrite/ferritecore/catalog/ferrite_mn-zn_material_characteristics_en.pdf

[27] Kemet Electronics Corporation, "Film capacitor datasheet". [Online]. Available: https://www.kemet.com/en/us/capacitors/film.html.

[28] OSCO Ltd, "Litz wire datasheet", 2021. [Online]. Available: http://www.osco.uk.com/products/cable-litz-wire.

[29] P. R. U. Guazzelli et al., "Weighting Factors Optimization of Predictive Torque Control of Induction Motor by Multi-objective Genetic Algorithm," IEEE Trans. Power Electron., vol. 34, no. 7, pp. 6628-6638, Jul. 2019.

[30] SAE International, "SAE J2954 - Wireless Power Transfer for Light-Duty Plugin/Electric Vehicles and Alignment Methodology," Warrendale, USA, 2019.

[31] Zhang Y et al., "A review of foreign object detection (FOD) for inductive power transfer systems, " in eTransportation, Jul. 2019.

[32] A. Zaheer et al., "Investigation of Multiple Decoupled Coil Primary Pad Topologies in Lumped IPT Systems for Interoperable Electric Vehicle Charging," IEEE Trans. Power Electron., vol. 30, no. 4, pp. 1937-1955, Apr. 2015.

[33] V. Cirimele, et al., "Challenges in the Electromagnetic Modeling of Road Embedded Wireless Power Transfer,” Energies, vol. 12, no. 14, p. 2677, Jul. 2019.

[34] V. Cirimele, M. Diana, F. Freschi and M. Mitolo, "Inductive Power Transfer for Automotive Applications: State-of-the-Art and Future Trends," in IEEE Transactions on Industry Applications, vol. 54, no. 5, pp. 4069-4079, Sept.-Oct. 2018. 